\title{
Myocarditis in COVID-19 patients: current problems
}

\author{
Donato Mele ${ }^{1}\left(\right.$ Filippo Flamigni $^{1} \cdot$ Claudio Rapezzi $^{1,2} \cdot$ Roberto Ferrari $^{1,2}$
}

Received: 20 July 2020 / Accepted: 6 January 2021 / Published online: 23 January 2021

(c) Società Italiana di Medicina Interna (SIMI) 2021

\begin{abstract}
Myocarditis has been reported as a possible clinical presentation or complication in patients with coronavirus disease (COVID)-19 due to SARS-CoV-2. Despite the alarm that this possibility generated among physicians, there is paucity of information about mechanisms, prevalence, prognosis, diagnosis and therapy of myocarditis in the context of COVID-19. This brief review has the goal to revise and summarize current knowledge on myocarditis in COVID-19 patients and underline problems especially related to diagnosis and treatment.
\end{abstract}

Keywords Myocarditis · Endotheliitis · COVID-19 · SARS-CoV-2

\section{Introduction}

The pandemic of SARS-CoV-2, known as coronavirus disease (COVID)-19, is currently a worldwide public health emergency. Interstitial pneumonia is the typical manifestation of the disease [1], which in some patients can bring to dramatic and lethal complications, such as diffuse inflammatory syndrome, multi-organ failure and disseminated intravascular coagulation. In many patients, cardiovascular manifestations have also been described [2]. In particular, after the publication of some case reports, the possibility of developing myocarditis and vasculitis has recently emerged. Myocarditis evocates well-known deadly complications [3], thus preoccupation developed among physicians and in the public opinion about the occurrence of heart inflammation [4]. Unfortunately, there is paucity of information on how to deal with diagnosis of myocarditis during COVID-19. Also, data on prevalence and prognosis are scarce and it is unclear whether myocarditis is an indirect complication of the disease or a direct cardiac manifestation of the virus [2]. This review has the goal to face and clarify these issues.

Donato Mele

donatomele@libero.it

1 Cardiology Center, University of Ferrara, Viale Aldo Moro 8, 44024 Cona, Ferrara, Italy

2 Maria Cecilia Hospital, GVM Care \& Research, Cotignola, Ravenna, Italy

\section{Diagnosis of myocarditis: which criteria?}

Diagnosis of myocarditis can be difficult in current clinical practice. A 2013 position paper from the Working Group on Myocardial and Pericardial Diseases of the European Society of Cardiology (ESC) [5] distinguishes clinically suspected and defined myocarditis. The clinical suspicious of myocarditis is based on patient clinics (mainly chest pain), electrocardiogram (ECG) (often elevation of the ST-segment), laboratory testing (ie, increase of serum troponin) and imaging findings, including those obtained using echocardiography and cardiac magnetic resonance (CMR). The CMR diagnostic criteria have been recently updated and include the coexistence of T1- and T2-related criteria indicating myocardial inflammation [6]. Beside the identification of myocardial damage, a fundamental step in the diagnostic process of myocarditis is the exclusion of obstructive coronary artery disease, especially when the clinical presentation resembles to an acute coronary syndrome [5]. However, even after coronary artery obstruction exclusion, diagnosis of myocarditis can remain doubtful, because other ischemic and non-ischemic diseases can have similar clinical features and non-obstructed coronary arteries [7]. Thus, definite diagnosis of myocarditis may require endomyocardial biopsy (EMB), which is still the gold standard evaluation [5]. EMB also allows the etiological diagnosis, for example the identification in the myocardium of a specific virus in presence of viral myocarditis $[5,8]$.

Despite EMB can bring to a definite diagnosis of myocarditis, in clinical practice, it is generally not performed 
in stable patients without heart failure and/or ventricular arrhythmias, especially in young low-risk patients. Also, because EMB execution requires a specific expertise, sometimes the patient needs to be transferred to a tertiary center for performing the procedure. A consensus paper from the American Heart Association (AHA)/American College of Cardiology (ACC) [9] indicates EMB as a class I procedure only in new onset heart failure with hemodynamic instability to exclude the presence of giant-cell, eosinophilic and hypersensitive myocarditis, because all these pathologies have a poor prognosis if untreated but they will dramatically improve if the specific therapy is done.

\section{Diagnosis of myocarditis in COVID-19 patients}

In patients with COVID-19, the criteria for myocarditis diagnosis are the same as in the other patients. However, the diagnostic pathway may be different, because it is conditioned, first of all, by the need to protect all health care operators from the risk of SARS-CoV-2 infection. Second, there may be differences related to the stable or unstable clinical status of the patient.

In critically ill patients admitted to intensive care units for whom myocarditis is clinically suspected, transportation to the radiology section or the cardiac catheterization laboratory can be problematic both for the difficulties related to intubation and to the possible electrical/hemodynamic instability. An accepted reason to bring an unstable COVID19 patient to the cardiac catheterization laboratory is the occurrence of acute ST-elevation myocardial infarction to be treated with percutaneous coronary intervention. In this setting, if there is no evidence of coronary obstruction and the suspicious of myocarditis arises, the EMB can be performed with no additional risk for the operators, provided that the expertise for the EMB procedure exists.

In clinically stable patients, both CMR and coronary computed tomography (CCT) could be theoretically performed for myocarditis diagnosis in a radiology section dedicated to COVID-19 patients. Such a radiology section is available, today, in many of the so-called COVID hospitals. In case a diagnostic confirmation is needed and there is local expertise for EMB, this could potentially be performed. However, most centers do not have local expertise for the EMB procedure. In this case, transferring a stable patient to a tertiary center for this procedure could be hazardous, because it could increase diffusion of the infection.

In both stable and unstable patients with clinically suspected myocarditis, transthoracic echocardiography is the first imaging technique generally performed at patient bedside and can be coupled with pulmonary ultrasound evaluation [10]. This technique, however, has limitations for myocarditis diagnosis. First, both global and regional ventricular systolic dysfunctions are not specific markers of acute myocarditis: ventricular dysfunction, in fact, can be due to a number of other cardiac diseases, of both ischemic and non-ischemic etiology [7]. On the other hand, patients with myocarditis can have a normal left ventricular ejection fraction (LV-EF), which, therefore, does not exclude this disease. In addition, the possibility of a pre-existing ventricular dysfunction should be always taken into account, especially if the patient has known cardiovascular risk factors but no documentation relative to recent ECGs or cardiac imaging examinations. Finally, it should be underlined that echocardiography can be problematic not only in patients intubated or admitted to the intensive care units because of low image quality but in general in COVID-19 patients. To reduce the operator exposure to potential infection, echocardiographic examinations are generally limited to fast collection of strictly necessary images. All these limitations should be well known to clinicians who are taking care of COVID-19 patients when they ask for an echocardiographic evaluation.

\section{Prevalence, characteristics, and prognosis of myocarditis in COVID-19 patients}

Usually, cardiovascular involvement in COVID-19 patients has been described as myocardial injury identified by troponin increase [11-14], thus a precise morphological and functional characterization of the heart in COVID-19 patients with clinically suspected myocarditis is lacking on a large scale. Nearly all the information about myocarditis in these patients comes from case reports or small series [15-21], which have been included in 3 systematic reviews so far [15-17].

Sawalha et al. [15] identified a total of 14 cases with myocarditis/myopericarditis believed to have occurred secondary to COVID-19 infection from December 1st 2019 to June 30th 2020 . There was a male predominance $(58 \%)$, with the median age of 50.4 years. The majority of patients did not have a previously identified comorbid condition (50\%); in those patients with a past medical history, hypertension was the most prevalent comorbidity (33\%). Of the 11 cases with documented hemodynamic status, the majority were in shock (64\%, 71\% cardiogenic, and 29\% mixed cardiogenic and septic shock). ECG findings were variable, and troponin was elevated in $91 \%$ of cases. Echocardiography was performed in most cases $(83 \%)$ and $60 \%$ had reduced LV-EF. Cardiac tamponade physiology was reported in $20 \%$ of all echocardiograms.

Kariyanna et al. [16] included 11 articles in their review. Nine were case reports and two were retrospective studies (all but one were peer-reviewed). Among the nine case reports, COVID-19 patients with myocarditis were $51.8 \pm 16.9$ years of age, and males and females were equally affected. In contrast, the retrospective studies described the typical myocarditis patient as male and older than 55 years 
of age [16]. The predominant complaint on presentation was dyspnea, followed by coughing, fever, and chest pain. All studies reported either increased troponin-I or T, along with elevated creatinine kinase-myocardial band (CK-MB) and brain natriuretic peptide (BNP). ECGs revealed ST-segment elevation in five cases, and inverted $\mathrm{T}$ waves in three cases. In six of the studies, echocardiography revealed decreased LV-EF.

Ho et al. [17] considered 24 case reports, 5 case series and 2 cohort studies, for a total of 31 publications on 51 patients; 12 cases were confirmed myocarditis while 39 had possible myocarditis. The median age was 55 years and $69 \%$ of patients were male. The most common presenting symptoms were fever, shortness of breath, cough, and chest pain. ECG changes included non-specific ST-segment and T-wave changes and ventricular tachycardia. Most patients had elevated cardiac and inflammatory biomarkers. LV systolic dysfunction and hypokinesis were common.

Regarding the diagnostic work-up for coronary artery disease, the report of Sawalha et al. [15] included CCT (17\% of cases) and invasive coronary angiography (25\% of cases). No patients were found to have any obstructive coronary disease. In the review of Karyianna et al. [16] coronary artery stenosis was not appreciated on the CCT studies.

Cardiac magnetic resonance was performed in $43 \%$ of cases in the report of Sawalha et al. [15] and showed diffuse late gadolinium enhancement (LGE). In the patients of Karyianna et al. [16] CMR revealed LGE in all of the patients, while myocardial edema was identified in more than half of the images. Fifty percent of the imaging studies revealed cardiomegaly or vascular redistribution. In the review of Ho et al. [17], CMR established the diagnosis in ten patients, with features of cardiac edema and cardiac injury.

Recently, Esposito et al. [18] reported the first series of ten patients with COVID-19 consecutively referred for CMR for suspected myocarditis between March 15 and April 20, 2020, in four Italian university hospitals. Cine images revealed normal LV end-diastolic volume in all patients (median $67 \mathrm{ml} / \mathrm{m}^{2}$; normal value $<90 \mathrm{ml} / \mathrm{m}^{2}$ ). Two patients were considered to have a takotsubo cardiomyopathy. For the remaining eight patients (six females and two males; $50 \pm 14$ years of age) the diagnosis of acute myocarditis was made. Five patients had preserved LV-EF (>55\%), and three patients had mildly reduced LV-EF (40\% to 55\%). In all cases, CMR showed diffuse intense myocardial edema. Surprisingly, LGE images were unremarkable and completely negative in five of eight patients. According with the authors' interpretation [18], the absence or very minimal amount of LGE observed in these patients suggest that diffuse edema can be the only CMR hallmark of myocardial inflammation in COVID-19, revealing unremarkable myocyte necrosis. This agrees with some histological results reporting limited or absent myocyte necrosis [22, 23], and may indicate an indirect mechanism causing myocardial inflammation.

Another important issue is the outcome of COVID-19 patients with clinically suspected and established myocarditis. Information can be extracted from the reviews mentioned above. In the review of Sawalha et al. [15], the majority of patients had survived to discharge (81\% of those with reported outcomes) with only a minority of cases not surviving (19\% of those with reported outcomes). All of the patients that reportedly passed away were noted to have both acute respiratory distress syndrome and myocarditis. Karyianna et al. [16] in their review reported only one death, and it was non-myocarditis-related; the majority of patients had either recovery or no further impairment of LV-EF. In the review of Ho et al. [17], although patients had complications such as heart failure, respiratory failure and multi-organ dysfunction, recovery of cardiac function occurred in three COVID-19 patients after severe disease requiring admission to the intensive care unit. Six patients recovered or were discharged, one showed improvement and two patients died. Esposito et al. [18] in their series observed that all patients were discharged at the time of writing, with regression of cardiac injury markers and LV functional recovery.

These observations evidence that most patients with clinically suspected myocarditis have a favorable in-hospital outcome and are discharged. Previously, one study hypothesized that myocarditis is responsible for the unfavorable outcome in many patients who died with cardiogenic shock [24]. This, however, needs confirmation, because it is unknown how the diagnosis of myocarditis was made in that investigation. It should also be underlined the complexity of the COVID-19 and the possibility to die for reasons not necessarily related to the initial cardiac involvement and presentation [25]. Finally, there is the need for follow-up studies to clarify what is the outcome of patients with the clinically suspected and established COVID-19 myocarditis after hospital discharge [26].

\section{Is myocarditis indirectly associated or directly due to SARS-CoV-2 infection?}

In a patient with COVID-19 and clinically suspected myocarditis in whom the EMB was performed, presence of cardiac lymphocytic infiltrates was documented but there was no evidence of intracellular viral material [22]. Huge interstitial edema and limited foci of necrosis were noted. Another EBM in a patient who died with cardiogenic shock showed low-grade interstitial and endocardial inflammation, presence of virus particles in cardiac macrophages but no viral trace in cardiomyocytes and endothelia and no signs of myocardial necrosis [23]. One hundred and four patients underwent EMB between February 3rd and March 26th, 2020, in German clinical centers for suspected myocarditis 
or unexplained heart failure [27]. In five cases, the SARSCoV-2 genome was detected by reverse transcriptase polymerase chain reaction. It was not possible, however, to determine the exact cell type that was susceptible to SARS-CoV-2 infection within the myocardium. Histopathological changes in myocardial tissue showed that the inflammatory process seemed to permeate vascular wall leading to small arterial obliteration and damage.

In an international multicenter study, autopsies were performed in 21 patients with COVID-19 and multifocal lymphocytic myocarditis was observed in 3 cases (14\%) [28]. Various forms of myocardial injury were also observed, including widespread increased interstitial macrophage infiltration in the myocardium ( $86 \%$ of cases) without clearly associated myocyte injury involving both the left and right ventricles. These authors did not report presence of virus particles in cardiac macrophages on electron microscopy. Molecular analysis for virus in the myocardium was not performed. In an autopsy study from Germany on 39 consecutive COVID-19 patients without fulminant myocarditis examined between April 8 and April 18, 2020, presence of SARS-CoV-2 within the myocardium was documented [29]. However, the most likely localization of virus was not in the cardiomyocytes but in interstitial cells or macrophages invading the myocardial tissue. Importantly, virus presence was not associated with increased infiltration of mononuclear cells into the myocardium and no myocarditis was present (because no massive cell infiltrates or necrosis could be documented). In another series of 12 consecutive autopsies from Germany (Hamburg) lymphocytic myocarditis was seen in the right ventricle of 1 patient (8.3\%) and viral RNA was also detected in the heart [30]. In a study from Buja et al. [31], who reviewed the pathological findings from initial published autopsy reports on 23 patients with COVID-19 from 5 centers in the United States of America, lymphocytic myocarditis was reported in 1 case (4.3\%). In 10 autopsies of African American decedents aged 44-78 years with cause of death attributed to COVID-19 in New Orleans, cardiac findings included individual cell necrosis (no large areas of myocyte necrosis) without lymphocytic myocarditis [32]. There was no obvious viral cytopathic effect by light microscopy, although the authors recognize that direct viral infection of myocytes could not be ruled out in their limited examination. In a series of 21 COVID-19 patients who underwent autopsy in Switzerland no signs of myocarditis were found [33].

A previously healthy 17-year-old African American male died on arrival to hospital after complaining of severe headaches, dizziness, nausea and vomiting for 2 days [34]. Post-mortem nasopharyngeal swabs detected SARS-CoV-2. Pathological examination of the heart found diffuse inflammatory infiltrates composed of lymphocytes, macrophages and prominent eosinophils, mainly in the interstitium and associated with multiple foci of myocyte necrosis. This was identified in both ventricles, and eosinophilic myocarditis was diagnosed. No other cause for the eosinophilic myocarditis was elucidated.

Overall, on the basis of the reports above, although myocarditis has been clearly documented at EBM or autopsy in some patients with COVID-19, there is no current evidence of myocarditis directly produced by the SARS-CoV-2 in humans. The associated lymphocytic myocarditis observed in patients with COVID-19 has been related to the generalized inflammatory reaction induced by cytokines [22]. The presence of virus particles in cardiac macrophages has been interpreted as the result of a viremic phase or, alternatively, the migration of infected alveolar macrophages in extra-pulmonary tissues [24]. As far as the eosinophilic myocarditis is concerned, it has been considered the possibility of an independent idiopathic eosinophilic myocarditis in which the stress of the COVID-19 contributed to the cardiac decompensation [34]. In the genesis of myocardial inflammatory findings at autopsy of patients with COVID-19 who died after potentially cardiotoxic anti-viral therapies, a druginduced myocarditis should also be considered $[35,36]$.

Some authors demonstrated a diffuse vasculitis directly produced by the SARS-CoV-2 [37]. They hypothesized that endotheliitis could explain the multi-organ impairment due to microvascular dysfunction in COVID-19 patients. According with these findings, it can also be speculated that cardiac vasculitis could induce myocardial impairment through microvascular dysfunction.

It should be emphasized that the EBM and autopsy data reported above were obtained only in a small number of patients with COVID-19. The majority of COVID-19 patients with a highly suggestive diagnosis of myocarditis did not receive a EMB confirmation. Post-mortem confirmation is also lacking in most cases, especially because autopsies in COVID-19 patients tend to be limited strictly to those cases for whom the cause of death needs clarification.

\section{Implications for therapy}

Therapy of myocarditis has not much evidence, since up to now only limited clinical trials were performed. Treatment should essentially be driven by the diagnosis of ventricular dysfunction and the definition of the etiology of the myocarditis. In particular, non-viral etiology may benefit from immunosuppressive therapy with a dramatic improvement of prognosis [38-41]. In viral myocarditis, treatment relates to viral isolation at EMB since some viruses have specific anti-viral therapy and some viral myocardites can improve with immunosuppressive therapy $[42,43]$.

Nonsteroidal anti-inflammatory drugs are generally not suggested in patients with myocarditis because they may cause renal impairment and sodium retention, which could further deteriorate acute ventricular dysfunction [5, 44]. In 
patients with fulminant myocarditis, a statement of the AHA recommends implementing the initial management protocol for cardiogenic shock, including administration of inotropes and/or vasopressors and mechanical ventilation [44]. Longer term management may require mechanical circulatory support [44].

In the specific case of myocarditis associated with COVID-19, some authors suggested to use high-dose steroids and intravenous immunoglobulins (IVIG) [13], driven by the idea that in late stage of COVID-19 there is a diffuse non-specific immune activation [45] However, the use of high-dose steroids in COVID-19 patients has given conflicting results. In one retrospective study, there was an improvement of survival [46], but another investigation showed a reduction in viral clearance, increased risk of sovrainfection and an increased mortality for all causes [47]. Regarding purified IVIG, there is supportive evidence for their use in acute myocarditis [48]. They gave encouraging result in a small group of five critical COVID-19 patients without clinically suspected myocarditis [49] but no additional evidence exists in patients with COVID-19 established myocarditis. The immunomodulatory effects of IVIG are multifactorial, because they showed not only anti-viral effects, but also antiinflammatory effects by suppressing inflammatory cytokines [50].

Different anti-viral agents were expected to be effective in patients hospitalized with COVID-19: remdesivir, hydroxychloroquine, lopinavir/ritonavir, and interferon beta1a. Unfortunately, all these drugs had little or no effect on overall mortality, initiation of ventilation, and duration of hospital stay [51, 52]. Therefore, data of efficacy of anti-viral agents in the setting of COVID-19 myocarditis are lacking.

The hypothesis of SARS-CoV-2 endotheliitis as the cause of systemic impaired microcirculatory function provides a rationale for therapies to stabilize the endothelium while tackling viral replication, particularly with anti-inflammatory anti-cytokine drugs, angiotensin converting enzyme inhibitors, and statins [53, 54]. In theory, this strategy could be particularly relevant for patients with pre-existing endothelial dysfunction, which is associated with several cardiovascular risk factors and established cardiovascular disease. This is, however, just speculative at the moment.

Recently, Siripanthong et al. [55] suggested a protocol for management of COVID-19-related myocarditis and arrhythmias, which are not uncommon in COVID-19 patients.

\section{A proposal for the diagnostic process in clinical practice}

If the suspicion of myocarditis arises in a patient with COVID-19 because of acute-onset cardiac symptoms or ECG modifications, both cardiac troponin and bedside echocardiography [56] should be obtained. The same applies for patients who develop electrical or hemodynamic instability. Particular attention should be given to biomarkers changes or trends rather than to values obtained in isolation. Subsequent invasive/non-invasive diagnostic work-up, including CMR [56] and CCT, may depend on the results of laboratory and echocardiography testing and on the clinical status of the patient. Main differential diagnoses are stress-induced cardiomyopathy, sepsis-related cardiomyopathy, and acute coronary syndrome, especially for the fulminant form of myocarditis [55].

\section{Conclusions}

Although cardiac inflammation has been documented in patients with COVID-19, at the moment there is no evidence of SARS-CoV-2 in human cardiomyocites, thus the possibility of a direct myocardial infection from the virus remains to be demonstrated. Treatment of clinically suspected myocarditis should currently be based on therapy for ventricular dysfunction and clinical status, including heart failure and cardiogenic shock. Evidence for the use of highdose steroids and IVIG is only anecdotal and does not allow systematic utilization of these drugs. Studies are needed to clarify the post-discharge outcome of clinically suspected myocarditis in COVID-19 patients, which at present has an indetermined incidence but seems uncommon. Finally, both clinicians and investigators should be very careful in distinguishing between clinically suspected and defined myocarditis to avoid misinterpretations and inaccurate estimations of myocarditis incidence in COVID-19 patients.

Author contributions DM wrote the first draft of the manuscript; FF, $\mathrm{CR}, \mathrm{RF}$ revised and approved the manuscript.

\section{Compliance with ethical standards}

Conflict of interest The authors declare that they have no conflict of interest.

Statement of human and animal rights This article does not contain any studies with human participants or animals performed by the authors.

Informed consent For this type of study, formal consent is not required.

\section{References}

1. Chen N, Zhou M, Dong X et al (2020) Epidemiological and clinical characteristics of 99 cases of 2019 novel coronavirus pneumonia in Wuhan, China: a descriptive study. Lancet 395:507-513 
2. Hendren NS, Drazner MH, Bozkurt B, Cooper LT Jr (2020) Description and proposed management of the acute COVID-19 cardiovascular syndrome. Circulation 141:1903-1914

3. Ali-Ahmed F, Dalgaard F, Al-Khatib SM (2020) Sudden cardiac death in patients with myocarditis: evaluation, risk stratification, and management. Am Heart J 220:29-40

4. https://www.nytimes.com/2020/03/27/health/coronavirus-cardi ac-heart-attacks.html

5. Caforio ALP, Pankuweit S, Arbustini E et al (2013) Current state of knowledge on aetiology, diagnosis, management, and therapy of myocarditis: a position statement of the European Society of Cardiology Working Group on Myocardial and Pericardial Diseases. Eur Heart J 34:2636-2648

6. Ferreira VM, Schulz-Menger J, Holmvang G et al (2018) Cardiovascular magnetic resonance in nonischemic myocardial inflammation: expert reccomendations. J Am CollCardiol 24:3158-3176

7. Thygesen K, Alpert JS, Jaffe AS, Chaitman BR et al (2019) Fourth universal definition of myocardial infarction. Eur Heart J 40:237-269

8. Leone O, Pieroni M, Rapezzi C, Olivotto I (2019) The spectrum of myocarditis: from pathology to the clinics. Virchows Arch 475:279-301

9. Cooper LT, Baughman KL, Feldman AM et al (2007) The role of endomyocardial biopsy in the management of cardiovascular disease: a scientific statement from American Heart Association, the American College of Cardiology, and the European Society of Cardiology. Circulation 116:2216-2233

10. D'Andrea A, Di Giannuario G, Marrazzo G et al (2020) The role of multimodality imaging in COVID-19 patients: from diagnosis to clinical monitoring and prognosis. G Ital Cardiol 21:345-353

11. Bonow RO, Fonarrow GC, O'Gara PT, Yancy CW (2020) Association of coronavirus disease 2019 (COVID-19) with myocardial injury and mortality. JAMA Cardiol 5:751-753

12. Yang X, Yu Y, Xu J et al (2020) Clinical course and outcomes of critically ill patients with SARS-CoV-2 pneumonia in Wuhan, China: a single-centered, retrospective, observational study. Lancet Respir Med 8:475-481

13. Shi S, Qin M, Shen B et al (2020) Association of cardiac injury with mortality in hospitalized patients with COVID-19 in Wuhan. China JAMA Cardiol 5:802-810

14. Zhou F, Yu T, Du R et al (2020) Clinical Course and risk factors for mortality of adult inpatients with COVID-19 in Wuhan, China: a retrospective cohort study. Lancet 395:1054-1062

15. Sawalha K, Abozenah M, Kadado AJ et al (2020) Systematic review of COVID-19 related myocarditis: insights on management and outcome. Cardiovasc Revasc Med. https://doi.org/10.1016/j. carrev.2020.08.028

16. Kariyanna PT, Sutarjono B, Grewal E et al (2020) A systematic review of COVID-19 and myocarditis. Am J Med Case Rep 8:299-305

17. Ho JS, Sia CH, Chan MY, Lin W, Wong RC (2020) Coronavirus-induced myocarditis: a meta-summary of cases. Heart Lung 49:681-685

18. Esposito A, Palmisano A, Natale L et al (2020) Cardiac magnetic resonance characterization of myocarditis-like acute cardiac syndrome in COVID-19. JACC Cardiovasc Imaging 13:2462-2465

19. Mele D, D'Andrea A, Campana $M$ et al (2020) Non-ischemic ventricular dysfunction in COVID-19 patients: characteristics and implications for cardiac imaging on the basis of current evidence. G Ital Cardiol 21:739-749

20. Khatri A, Wallach F (2020) Coronavirus disease 2019 (Covid19) presenting as purulent fulminant myopericarditis and cardiac tamponade: a case report and literature review. Heart Lung 49:858-863

21. Ozieranski K, Tyminska A, Jonik S et al (2020) Clinically suspected myocarditis in the course of severe acute respiratory syndrome novel coronavirus-2 infection: fact or fiction? J Cardiac Fail. https://doi.org/10.1016/j.cardfail.2020.11.002

22. Sala S, Peretto G, Gramegna M et al (2020) Acute myocarditis presenting as a reverse Tako-Tsubo syndrome in a patient with SARS-CoV-2 respiratory infection. Eur Heart J 41:1861-1862

23. Tavazzi G, Pellegrini C, Maurelli M et al (2020) Myocardial localization of coronavirus in COVID-19 cardiogenic shock. Eur J Heart Fail 22:911-915

24. Ruan Q, Yang K, Wang W, Jiang L, Song J (2020) Clinical predictors of mortality due to COVID-19 based on an analysis of data of 150 patients from Wuhan. Intensive Care Med 46:846-848

25. Zeng J-H, Liu Y-X, Yuan J et al (2020) First case of COVID19 complicated with fulminant myocarditis: a case report and insights. Infection 48:773-777

26. Shchedrygina A, Nagel E, Puntmann VO, Valbuena-Lopez S (2020) COVID-19 myocarditis and prospective heart failure burden. Expert Rev Cardiovasc Ther. https://doi.org/10.1080/14779 072.2021 .1844005

27. Escher F, Pietsch H, Aleshcheva G et al (2020) Detection of viral SARS-CoV-2 genomes and histopathological changes in endomyocardial biopsies. ESC Heart Failure 7:2440-2447

28. Basso C, Leone O, Rizzo S et al (2020) Pathological features of COVID-19-associated myocardial injury: a multicentre cardiovascular pathology study. Eur Heart J 41:3827-3835

29. Lindner D, Fitzek A, Bräuninger $\mathrm{H}$ et al (2020) Association of cardiac infection with SARS-CoV-2 in confirmed COVID-19 autopsy cases. JAMA Cardiol 5:1281-1285

30. Wichmann D, Sperhake J-P, Lutgehetmann M et al (2020) Autopsy findings and venous thromboembolism in patients with COVID-19 a prospective cohort study. Ann Intern Med. https:// doi.org/10.7326/M20-2003

31. Buja LM, Wolf DA, Zhao B et al (2020) The emerging spectrum of cardiopulmonary pathology of the coronavirus disease 2019 (COVID-19): Report of 3 autopsies from Houston, Texas, and review of autopsy findings from other United States cities. Cardiovascular Pathology 48:107233

32. Fox SE, Akmatbekov A, Harbert JL et al (2020) Pulmonary and cardiac pathology in African American patients with COVID19: an autopsy series from New Orleans. Lancet Respir Med 8:681-686

33. Menter T, Haslbauer JD, Nienhold R et al (2020) Postmortem examination of COVID-19 patients reveals diffuse alveolar damage with severe capillary congestion and variegated findings in lungs and other organs suggesting vascular dysfunction. Histopathology 77:198-209

34. Craver R, Huber S, Sandomirsky M et al (2020) Fatal eosinophilic myocarditis in a healthy 17-year-old male with severe acute respiratory syndrome coronavirus 2 (SARS-CoV-2c). Fetal Pediatr Pathol 39:263-268

35. Russell B, Moss C, George G et al (2020) Associations between immune-suppressive and stimulating drugs and novel COVID19-a systematic review of current evidence. Ecancermedicalscience 14:1022

36. Alijotas-Reiga J, Esteve-Valverde E, Beliznaf C et al (2020) Immunomodulatory therapy for the management of severe COVID-Beyond the anti-viral therapy: a comprehensive review. Autoimmunity Rev 19:102569

37. Varga Z, Flammer AJ, Steiger P et al (2020) Endothelial cell infection and endotheliitis in COVID-19. Lancet 395:1417-1418

38. Cooper LT, Berry GJ, Shabetai R (1997) Idiopathic giantcell myocarditis-natural history and treatment. Multicenter giant cell myocarditis study group investigators. N Engl J Med 336:1860-1866

39. Cooper LT Jr, Hare JM, Tazelaar HD et al (2008) Giant cell myocarditis treatment trial investigators usefulness of immunosuppression for giant cell myocarditis. Am J Cardiol 102:1535-1539 
40. Frustaci A, Chimenti C, Calabrese F et al (2003) Immunosuppressive therapy for active lymphocytic myocarditis: virological and immunologic profile of responders versus nonresponders. Circulation 107:857-863

41. Frustaci A, Russo MA, Chimenti C (2009) Randomized study on the efficacy of immunosuppressive therapy in patients with virus-negative inflammatory cardiomyopathy: the TIMIC study. Eur Heart J 30:1995-2002

42. Mason JW, Billingham ME, Ricci DR (1980) Treatment of acute inflammatory myocarditis assisted by endomyocardial biopsy. Am J Cardiol 45:1037-1044

43. Jones SR, Herskowitz A, Hutchins GM, Baughman KL (1991) Effects of immunosuppressive therapy in biopsy-proved myocarditis and borderline myocarditis on left ventricular function. Am J Cardiol 68:370-376

44. Kociol RD, Cooper LT, Fang JC et al (2020) Recognition and initial management of fulminant myocarditis: a scientific statement from the American Heart Association. Circulation 141:e69-e92

45. Mehta P, McAuley DF, Brown M et al (2020) COVID-19: consider cytokine storm syndromes and immunosuppression. Lancet 395:1033-1034

46. Wu C, Chen X, Cai Y et al (2020) Risk factors associated with acute respiratory distress syndrome and death in patients with coronavirus disease 2019 pneumonia in Wuhan. China JAMA Intern Med 180:934-943

47. Russell CD, Millar JE, Baillie JK (2020) Clinical evidence does not support corticosteroid treatment for 2019-nCoV lung injury. Lancet 395:473-475

48. Huang X, Sun Y, Su G, Li Y, Shuai X (2019) Intravenous immunoglobulin therapy for acute myocarditis in children and adults. Int Heart J 60:359-365

49. Shen C, Wang Z, Zhao F et al (2020) Treatment of 5 critically Ill patients with COVID-19 with convalescent plasma. JAMA 323:1582-1589
50. Kow CS, Hasan SS (2020) Glucocorticoid versus immunoglobulin in the treatment of COVID-19-associated fulminant myocarditis. Infection 48:805-806

51. WHO Solidarity Trial Consortium (2020) Repurposed antiviral drugs for COVID-19-interim WHO solidarity trial results. N Engl J Med. https://doi.org/10.1056/NEJMoa2023184

52. Recovery Collaborative Group (2020) Lopinavir-ritonavir in patients admitted to hospital with COVID-19 (RECOVERY): a randomised, controlled, open-label, plat-form trial. Lancet 396:1345-1352

53. Xu Z, Shi L, Wang Y et al (2020) Pathological findings of COVID-19 associated with acute respiratory distress syndrome. Lancet Respir Med 8:420-422

54. Evans PC, Rainger GE, Mason JC et al (2020) Endothelial dysfunction in COVID-19: a position paper of the ESC Working Group for Atherosclerosis and Vascular Biology, and the ESC Council of Basic Cardiovascular Science. Cardiovasc Res 116:2177-2184

55. Siripanthong B, Nazarian S, Muser D et al (2020) Recognizing COVID-19-related myocarditis: The possible pathophysiology and proposed guideline for diagnosis and management. Heart Rhythm 17:1463-1471

56. Cau R, Bassareo P, Saba L (2020) Cardiac involvement in COVID-19-assessment with echocardiography and cardiac magnetic resonance imaging. SN Compr Clin Med. https://doi. org/10.1007/s42399-020-00344-7

Publisher's Note Springer Nature remains neutral with regard to jurisdictional claims in published maps and institutional affiliations. 